\title{
NOTE ON THE DEGREE OF CONVERGENCE OF SEQUENCES OF POLYNOMIALS
}

\author{
J. L. WALSH AND E. N. NILSON
}

The object of this note is to establish the following result:

THEOREM. Let the power series

$$
f(z) \equiv \sum_{n=0}^{\infty} a_{n} z^{n}
$$

have the radius of convergence $\rho(>1)$, and let $p_{n}(z)$ denote the polynomial of degree $n$ of best approximation to $f(z)$ in the closed region $|z| \leqq 1$ in the sense of Tchebycheff. A necessary and sufficient condition for

$$
\lim _{n \rightarrow \infty}\left[\max \left|f(z)-p_{n}(z)\right|, \text { for }|z| \leqq 1\right]^{1 / n}=1 / \rho
$$

is that $f(z)$ not be of lacunary structure.

It is well known ${ }^{1}$ that the equation

$$
\limsup _{n \rightarrow \infty}\left[\max \left|f(z)-p_{n}(z)\right|, \text { for }|z| \leqq 1\right]^{1 / n}=1 / \rho
$$

is valid for every $f(z)$ defined by a power series as in (1) with radius of convergence $\rho$. The significance of the theorem is that the stronger relation (2) is valid except for functions of lacunary structure, as defined by Bourion. ${ }^{2}$

If and only if $f(z)$ is of lacunary structure, the partial sums $s_{n}(z) \equiv \sum_{0}^{n} a_{k} z^{k}$ are polynomials of degree $n$ of which a suitably chosen subsequence $s_{n_{k}}(z)$ has the property (Bourion, loc. cit.)

(4) $\quad \limsup _{n_{k} \rightarrow \infty}\left[\max \left|f(z)-s_{n_{k}}(z)\right| \text {, for }|z| \leqq r\right]^{1 / n_{k}}<r / \rho, 0<r<\rho^{\circ}$

If (4) holds for a single $r, 0<r<\rho$, it holds for every such $r$.

If $f(z)$ is of lacunary structure, then for the extremal polynomials $p_{n}(z)$ of best approximation we have

$$
\begin{aligned}
{\left[\max \left|f(z)-p_{n_{k}}(z)\right|,\right. \text { for }} & |z| \leqq 1] \\
& \leqq\left[\max \left|f(z)-s_{n_{k}}(z)\right|, \text { for }|z| \leqq 1\right],
\end{aligned}
$$

Received by the editors June 24, 1946.

1 Walsh, Interpolation and approximation by rational functions in the complex domain. Amer. Math. Soc. Colloquium Publications, vol. 20, 1935, chap. 4.

${ }^{2}$ L'ultraconvergence dans les séries de Taylor, Actualités Scientifiques et Industrielles, no. 472, 1937, pp. $9 \mathrm{ff}$. 
whence from (4)

$$
\underset{n_{k} \rightarrow \infty}{\lim \sup _{0}}\left[\max \left|f(z)-p_{n_{k}}(z)\right|, \text { for }|z| \leqq 1\right]^{1 / n_{k}}<1 / \rho,
$$

and (2) is not satisfied.

Conversely, if (2) is not satisfied, then for a suitably chosen sequence $n_{k}$ the first member of (5), which we denote by $1 / \rho^{\prime}$, is less than $1 / \rho$. For values of $z$ interior to the unit circle $C$ we have the relations

$$
\begin{gathered}
f(z)-s_{n}(z)=\frac{1}{2 \pi i} \int_{C} \frac{z^{n+1} f(t) d t}{t^{n+1}(t-z)}, \\
0=\frac{1}{2 \pi i} \int_{C} \frac{z^{n+1} p_{n}(t) d t}{t^{n+1}(t-z)}, \\
f(z)-s_{n}(z)=\frac{1}{2 \pi \imath} \int_{C} \frac{z^{n+1}\left[f(t)-p_{n}(t)\right] d t}{t^{n+1}(t-z)}, \\
\limsup _{n_{k} \rightarrow \infty}\left[\max \left|f(z)-s_{n k}(z)\right|, \text { for }|z| \leqq r<1\right]^{1 / n_{k}} \leqq r / \rho^{\prime}<r / \rho,
\end{gathered}
$$

whence $f(z)$ is of lacunary structure. The theorem is established.

The generalization of equation (2), where we now consider approximation on a suitably chosen more general point set than $|z| \leqq 1$, furnishes a generalization of the concept of functions of lacunary structure.

HaRvard University aNd

United States Naval Academy 